\title{
Fondos europeos para la competitividad y su absorción en las regiones españolas (2007-2015)
}

\section{Cristina García Nicolás', Encarnación Murillo García², Milagros Paniagua San Martín ${ }^{3}$}

Resumen: Frente al principio de cohesión, ligado al de solidaridad, los retos derivados de las ampliaciones y de la globalización económica han llevado a la Unión Europea a priorizar la competitividad en el diseño de la nueva política de cohesión. Tras un breve repaso a la evolución conceptual de los principios de convergencia-cohesión y de competitividad, el objetivo de este trabajo es analizar si en el caso de las regiones españolas el gasto ejecutado asociado a las ayudas estructurales europeas refleja la prioridad del objetivo de competitividad durante el periodo 2007-2015. Igualmente, se pretende observar si existe homogeneidad en esa ejecución entre diferentes tipologías de territorios, así como la capacidad de absorción de dichos fondos, es decir, si se ejecutan las partidas programadas para este concepto, en la misma media que las del conjunto de los programas confinanciados con ayudas europeas.

Palabras Clave: Cohesión, competitividad, política regional, programas operativos, innovación.

Fecha de recepción: I de septiembre de 2020.

Fecha de admisión definitiva: 18 de diciembre de 2020.

\footnotetext{
' Universidad de Castilla-La Mancha. cristina.garcia@uclm.es

${ }^{2}$ Universidad Rey Juan Carlos y EQUALITAS. encarnacion.murillo@uric.es

${ }^{3}$ Universidad Rey Juan Carlos.
} 
European funds for competitiveness and their allocation in spanish regions (2007-2015)

Abstract: The challenges arising from its expansion as well as economic globalisation have led the European Union to prioritise competitiveness rather than cohesion and solidarity in the design of its new cohesion policy. After a brief review of the conceptual evolution of the principles of convergence-cohesion and competitiveness, the aim of this paper is to analyse whether, in the case of the Spanish regions, the expenditure associated with European structural aid reflects the priority of the competitiveness objective during the 2007-2015 period. Likewise, it aims to observe whether allocation in different types of territories is homogeneous, and looks at the absorption capacity for these funds; that is, whether the items programmed for this concept are allocated in the same manner as those of all the programmes co-financed with European aid.

Key words: Cohesion, competitiveness, regional politics, operational programmes, innovation.
Fonds européens pour la compétitivité et leur allocation dans les régions espagnoles (2007-2015)

Résumé: Les défis découlant de son expansion ainsi que de la mondialisation économique ont conduit l'Union européenne à privilégier la compétitivité plutôt que la cohésion et la solidarité dans la conception de sa nouvelle politique de cohésion. Après un bref examen de l'évolution conceptuelle des principes de convergence-cohésion et de compétitivité, l'objectifde ce documentestd'analyser si, dans le cas des régions espagnoles, les dépenses associées aux aides structurelles européennes reflètent la priorité de l'objectif de compétitivité au cours de la période 2007-2015. De même, il vise à observer si l'allocation dans les différents types de territoires est homogène, et examine la capacitéd'absorption de ces fonds, c'est-à-dire si les éléments programmés pour ce concept sont alloués de la même manière que ceux de tous les programmes cofinancés avec l'aide européenne.

Mots clé: Cohésion, compétitivité, politique régionale, programmes opératives, innovation.

\section{Introducción}

Desde la entrada de España y Portugal en la entonces Comunidad Económica Europea y desde la firma del Acta Única (1986) que consolidó la política regional, no sólo se han alternado procesos de profundización político-económica con otros de ampliación, sino que se ha evolucionado desde la citada política regional hasta una política de cohesión, a la que el Tratado de Ámsterdam (1997) añadió su vertiente social, y el Tratado de Lisboa (2009) reforzó en lo territorial.

Este cambio de nomenclatura incluye también una modificación de sus objetivos, favorecida en buena medida por las transformaciones que, a todos los niveles, ha supuesto la globalización y la necesidad derivada de situar a la Unión Europea en ese escenario político y económico ampliado. En este contexto es preciso no olvidar 
que los ajustes y revisiones que se realizan durante este periodo de programación podrían estar relacionados con la Estrategia Europa 2020, que, presentada por la Comisión Europea en 2010, vincula la salida de la crisis económica de 2008 a un crecimiento que interrelaciona tres prioridades: que sea inteligente, sostenible e integrador. Es ahí donde la competitividad para afrontar los cambios en la globalización enlaza con los objetivos de la política de cohesión.

El lugar central que ocupa este objetivo de competitividad, en sus diversas acepciones, en la política de inversiones en la UE ha llevado a plantear este trabajo. Su principal objetivo es analizar el gasto asociado al objetivo de la competitividad durante el periodo 2007-2013 en el ámbito concreto de España y de sus Comunidades Autónomas. El marco cronológico se alarga hasta 2015 con el fin de acceder a los datos definitivos de aplicación y gasto de las partidas determinadas siguiendo la regla $\mathrm{n}+2^{4}$, lo que nos facilitará determinar la absorción de dichos fondos, comparando las partidas programadas con su aplicación efectiva. Para ello utilizaremos los datos territorializados de los Programas Operativos FEDER $y$, en concreto, las partidas correspondientes a l+D+i y a Pymes porque guardan una relación más estrecha con los principios de competitividad e innovación, que posteriormente se estudiarán a través de los correspondientes índices regionales y sus indicadores.

Para la realización de dicho análisis el trabajo se estructura de la siguiente manera: tras esta introducción, un segundo apartado aborda algunos de los principales elementos de la evolución de la terminología utilizada en el diseño de la política regional, así como de los principios aplicados en los últimos periodos de programación; el tercer apartado está dedicado a mostrar y analizar, a partir de los datos correspondientes a los Programas Operativos-FEDER, que el cambio teórico ya estudiado, se ve reflejado en las cuantías presupuestarias asignadas a este objetivo de competitividad y en su grado de ejecución. La composición y distribución de aquéllos se considerará para el caso español y sus regiones, y se complementará con un análisis del Índice regional de competitividad de 2016 y el Índice regional de innovación de 2017 que muestran la posición de España y de las Comunidades Autónomas en el contexto de la Unión Europea. El trabajo se cierra con las principales conclusiones del trabajo, y unas reflexiones finales.

${ }^{4}$ La regla $n+2$ es la norma financiera de la asignación presupuestaria anual de los Fondos Estructurales (FF.EE.) y el Fondo de Cohesión (FC) de la UE. Si los fondos no se han gastado en la fecha prevista, la Comisión puede liberar el compromiso de futuras asignaciones. La liberación se efectúa de oficio si los fondos no se han gastado, o no se han presentado solicitudes de pago, al finalizar el segundo año $(n+2)$. 


\section{La política de cohesión europea: marco de referencia, prin- cipios y objetivos}

Una de las consecuencias, o quizás también causa, del peso actual del principio de competitividad frente al de convergencia, podría ser, como indica Joven (201 1, p. 60) un progresivo divorcio entre el objetivo de cohesión y la políitica de desarrollo regional. En este apartado trataremos de mostrar si existen trayectorias divergentes que alejan a la política de cohesión del principio de solidaridad y la acercan más al de competitividad.

\section{I. Evolución del principio de convergencia}

Aunque desde la firma de los Tratados de Roma existe una preocupación constante por las disparidades regionales, habrá que esperar a la firma del Acta Única (1986) para que la política de cohesión económica y social reciba un mayor apoyo dedicándole la subsección IV de la Sección II. Igualmente, la política regional pasa a convertirse en uno de los más firmes pilares del principio de cohesión social comunitario, estableciendo como objetivos no sólo la aminoración de las disparidades regionales, sino también la creación en las regiones deprimidas de las condiciones económicas, sociales y culturales, que permitieran una convergencia efectiva de sus niveles de vida con el de las regiones más desarrolladas.

La reforma de los Fondos Estructurales en 1988 (Delors I) consolida una verdadera política regional comunitaria a partir de los principios de concentración, cooperación, programación y adicionalidad. Principios que se mantendrán en la reforma de 1993 (Delors II), en la que no se introducen reformas tan profundas en los Reglamentos. No obstante, se incorpora la definición de las "acciones innovadoras» (de acuerdo con el art. 10 del Reglamento CEE 2083/93 relativo al FEDER, y el Programa Europeo para Cooperación Interregional e Innovación económica regional) que incluyen nuevos conceptos en materia de ordenación del territorio, cooperación transfronteriza, redes de cooperación e intercambio de experiencias entre ciudades y regiones, o sobre cuestiones relacionadas con la ciudad. A todo lo anterior se suman las Iniciativas Comunitarias, destinadas a regiones con derecho a beneficiarse de los objetivos de la cohesión económica y social.

La incorporación de estas últimas responde a los objetivos del Tratado de la Unión Europea (TUE), firmado en Maastricht (1992), que hizo de la cohesión económica y social un objetivo prioritario de la Comunidad, en paralelo a la Unión Económica y Monetaria (UEM), y al mercado único. Cinco años después, el Tratado de 
Ámsterdam (1997) incluye en las normas constitucionales de la UE la problemática del empleo y de la política social solamente abordadas de forma limitada en Maastricht, suponiendo el principal giro social en todo el proceso de construcción comunitaria actual. Igualmente se incorpora el principio de la cooperación reforzada, regulándose con carácter general -ante la perspectiva de una Europa más amplia y diversa- la posibilidad de una integración diferenciada, en la que no todos los Estados avancen al mismo tiempo, poniendo en riesgo los principios de cohesión y solidaridad.

Este mismo año 1997 se inician los Informes sobre la cohesión, a través de los cuales es posible analizar no solo la evolución del concepto, sino las claves que regirán la política regional en cada uno de los periodos de programación (el último y VII Informe se presentó en 2017). A pesar de que todos hacen referencia a las disparidades regionales, el concepto de solidaridad-que aparecía en los arts. 2 y 3 del Tratado de Roma- sólo se menciona en el primero de ellos al definir el objetivo de la cohesión como "la puesta en práctica de los propósitos de solidaridad y apoyo mutuo a través de objetivos mensurables de carácter social y económico".

La adhesión de diez Estados en 2004 (completada en 2007 con la entrada de Rumanía y Bulgaria) constituirá el mayor reto del periodo de programación 2000-2006, con la incorporación de nuevas divergencias territoriales que desplazan el interés político y las necesidades socioeconómicas hacia el Este del continente.

En buena medida consecuencia de la ampliación anterior, el inicio del periodo de programación 2007-2013 viene marcado por la incorporación de la dimensión territorial al proceso de cohesión, reforzada por dos textos fundamentales: la Agenda Territorial y la Carta de Leipzig. La cohesión territorial, entendida como la distribución equilibrada de las actividades humanas en el territorio, representa la traslación en términos de territorio del objetivo de desarrollo sostenible y equilibrado asignado a la Unión (artículo 2 del Tratado). Ya había ido adquiriendo un progresivo reconocimiento institucional desde la primera mención en el Segundo Informe sobre la Cohesión hasta el Tratado por el que se establece una Constitución para Europa, en cuyo art. I.3 aparece como uno de los objetivos de la Unión. La modificación del Tratado en la Cumbre de Lisboa de 2007 -firmado el 13 de diciembre-, mantiene las referencias anteriores a la cohesión territorial e incorpora otras nuevas en la denominación del Título XVII que pasa a ser "Cohesión económica, social y territorial», además de incluir en el artículo 2, y dentro de los objetivos de la Unión Europea, el fomento de la cohesión social, económica y territorial, así como la solidaridad entre los Estados miembros. La dimensión territorial es fundamental si realmente quiere conseguirse la cohesión, y más en 
el contexto de un ámbito geográfico cada vez más extenso y diverso, tal como indicaba el Consejo en 2006: "contribuirá a desarrollar comunidades sostenibles y a evitar un desarrollo regional desequilibrado que obstaculice el potencial de crecimiento global" (Consejo Europeo, 2006, p. 29).

\subsection{El objetivo de competitividad}

Para entender la evolución de los objetivos de desarrollo (que podría ligarse al de competitividad) y de cohesión en la Unión Europea, es preciso hacer referencia a la Estrategia de Lisboa (2000), revitalizada en 2005 con la Estrategia Renovada de Lisboa. El Consejo Europeo de Lisboa (23 y 24 de marzo de 2000) aprobaba en sus Conclusiones un nuevo objetivo estratégico para la próxima década: "convertirse en la economía basada en el conocimiento más competitiva y dinámica del mundo, capaz de crecer económicamente de manera sostenible con más y mejores empleos y con mayor cohesión social". La sociedad de la información y de Investigación y Desarrollo (I+D), la inversión en capital humano (teniendo en cuenta que se acababa de poner en marcha el Espacio Europeo de Educación), y la lucha contra la exclusión social, junto con el pleno empleo, constituían objetivos demasiado ambiciosos que hicieron necesaria una redefinición de la estrategia, tal como se indicaba en el Informe Kok (2003). Así, la Estrategia renovada de Lisboa (2005) recogía parte de los retos planteados cinco años antes, estructurados en tres pilares con sus respectivos objetivos:

1. Un lugar más atractivo para invertir y trabajar. Objetivo que suponía importantes mejoras en las reglamentaciones europeas y nacionales, acompañadas del desarrollo del mercado interior y de las infraestructuras europeas, así como de la garantía de mercados abiertos y competitivos dentro y fuera de Europa.

2. El conocimiento y la innovación, factores de crecimiento. La realización de este objetivo implicaba, en primer lugar, aumentar y mejorar la inversión en investigación y desarrollo, para a continuación facilitar la innovación, la adopción de las tecnologías de la información y la comunicación y el uso sostenible de los recursos. Uno de los resultados de las dos primeras premisas sería la creación de una base industrial europea sólida.

3. Crear más empleo y de mejor calidad. Este fue uno de los grandes objetivos fallidos, de tal modo que en esta ocasión no se aspira al pleno empleo, sino a atraer a un mayor número de personas al mercado laboral, modernizando también los sistemas de protección social. Además, se considera la necesidad 
de invertir en la cualificación de los trabajadores, que habrán de ser capaces, junto con las empresas, de adaptarse a unos mercados laborales cada vez más flexibles.

En 2009 el Informe Barca propone una nueva política de cohesión que se apoyaba en dos conceptos clave, la eficiencia territorial y la equidad. Y es precisamente en la gestión donde la política de cohesión experimenta una importante evolución a lo largo de la primera década del siglo actual (Joven, 2011, pp. 65-66): en primer lugar, los gestores nacionales y regionales se convierten en los únicos interlocutores para los beneficiarios finales de la política europea de cohesión; en segundo lugar, se produce una simplificación de la puesta en práctica, tanto con respecto al número de objetivos, que se reduce de seis a tres, como en lo referido al gasto y su control; $y$, por último, se hace evidente la preeminencia de la competitividad sobre la solidaridad, vinculada a la Estrategia de Lisboa, que favorece que se ponga el acento en los resultados y en las inversiones regionales en los sectores más innovadores.

Además de la ya citada incorporación de la dimensión territorial de la cohesión, el periodo de programación 2007-2013 supone una reforma en profundidad de la arquitectura de la política regional comunitaria en objetivos, financiación y criterios de elegibilidad (Cuadro 1). Todo ello con el fin de conseguir una aproximación estratégica de la programación a las realidades nacionales, regionales y locales. Se establecen tres áreas prioritarias subvencionadas únicamente por dos Fondos Estructurales y el Fondo de Cohesión, y basadas en la "convergencia»-objetivo que atenderá el crecimiento y el empleo en las regiones menos desarrolladas, mediante proyectos de modernización y diversificación de la economía, el desarrollo de las infraestructuras y la mejora del sistema de formación y educación de cara al empleo-; la "competitividad»-aunque también abarca al resto de regiones no incluidas en el objetivo "convergencia», constituye un objetivo fundamentalmente nacional relacionado con la Estrategia Europea de Empleo y trabajará aspectos como la previsión en los cambios económicos y sociales, la lucha contra el paro, el apoyo a la innovación y a la sociedad del conocimiento, la prevención de riesgos, la mejora de la accesibilidad, la inclusión social, la formación continua, la igualdad de oportunidades y la lucha contra las discriminaciones-; y la "cooperación» - derivada de la experiencia de la Iniciativa Comunitaria Interreg, contribuirá a un desarrollo armonioso del territorio de la Unión Europea-. 


\section{CUADRO I. Cohesión 2007-2013: objetivos e instrumentos}

\begin{tabular}{|c|c|c|c|}
\hline \multicolumn{2}{|c|}{ 2000-2006 } & \multicolumn{2}{|c|}{ 2007-2013 } \\
\hline Objetivos & $\begin{array}{l}\text { Instrumentos } \\
\text { financieros }\end{array}$ & Objetivos & $\begin{array}{l}\text { Instrumentos } \\
\text { financieros }\end{array}$ \\
\hline Fondo de Cohesión & Fondo de Cohesión & \multirow{3}{*}{ Convergencia } & FEDER \\
\hline \multirow[b]{2}{*}{ Objetivo $n^{\circ} 1$} & \multirow{2}{*}{$\begin{array}{l}\text { FEDER } \\
\text { FSE } \\
\text { FEOGA-Garantía } \\
\text { FEOGA-Orientación } \\
\text { IFOP }\end{array}$} & & FSE \\
\hline & & & Fondo de Cohesión \\
\hline Objetivo $n^{\circ} 2$ & $\begin{array}{l}\text { FEDER } \\
\text { FSE }\end{array}$ & \multirow{2}{*}{$\begin{array}{l}\text { Competitividad } \\
\text { regional y empleo } \\
\text { Nivel regional } \\
\text { Nivel nacional: } \\
\text { estrategia europea } \\
\text { de empleo }\end{array}$} & \multirow[b]{2}{*}{$\begin{array}{l}\text { FEDER } \\
\text { FSE }\end{array}$} \\
\hline Objetivo $n^{\circ} 3$ & FSE & & \\
\hline Interreg & FEDER & \multirow{4}{*}{$\begin{array}{l}\text { Cooperación } \\
\text { territorial europea }\end{array}$} & \multirow{4}{*}{ FEDER } \\
\hline URBAN & FEDER & & \\
\hline EQUAL & FSE & & \\
\hline Leader+ & FEOGA-Orientación & & \\
\hline $\begin{array}{l}\text { Desarrollo rural y } \\
\text { reestructuración del } \\
\text { sector pesquero fuera } \\
\text { del objetivo } n^{\circ} 1\end{array}$ & $\begin{array}{l}\text { FEOGA-Garantía } \\
\text { IFOP }\end{array}$ & & \\
\hline 9 objetivos & 6 instrumentos & 3 objetivos & 3 instrumentos \\
\hline
\end{tabular}

Fuente: Unión Europea, 2004.

La simplificación se extiende tanto al número de objetivos y de instrumentos financieros como a la programación. Esta última se realizará ahora en una sola etapa, puesto que se han eliminado el Marco Comunitario de Apoyo y el Complemento de Programación ${ }^{5}$. El Marco Estratégico Nacional de Referencia (MENR) (arts. 27

${ }^{5}$ Los Marcos Comunitarios de Apoyo (MCA) son documentos de programación, aprobados por la Comisión Europea tras el análisis de los planes de desarrollo que presentan los Estados miembros. Describen la estrategia de desarrollo y las prioridades de actuación a cofinanciar con los recursos comunitarios, 
y 28 del Reglamento general) aporta una única estrategia respondiendo a la visión de conjunto de cada Estado miembro. Los campos de interés de la nueva política regional y de cohesión quedan reflejados en el reparto del presupuesto comunitario, correspondiendo en 2007 el $80 \%$ de los recursos a medio ambiente y cohesión.

En cuanto a las prioridades del Objetivo 1 -ahora, «Convergencia»-, correspondiente a las regiones menos desarrolladas, existen también diferencias con respecto al periodo 2000-2006. En primer lugar, desaparecen las relacionadas con agricultura y desarrollo rural, y pesca y acuicultura, como consecuencia de que el FEADER (Fondo Europeo Agrícola de Desarrollo Rural) y el FEP (Fondo Europeo de Pesca) están fuera de la política de cohesión. Se mantiene la prioridad de la sociedad de información, y del medio ambiente, hábitats naturales y recursos, complementada en el nuevo periodo por la prevención de riesgos. Se incorporan el desarrollo e innovación de empresas, y transporte y energía. Y se añade "sostenible" al desarrollo local y urbano, y "capacidad institucional" a la asistencia técnica. Los aspectos más sociales relacionados con el empleo, así como la igualdad de oportunidades son fundamentalmente objetivos del Fondo Social, aunque se incorpora la prioridad de infraestructuras sociales.

Por lo que se refiere al caso español, el MENR establece tres grandes objetivos estrechamente relacionados con la Estrategia renovada de Lisboa, tal como muestra el Cuadro 2. Estos Objetivos Finales se implementan a través de 27 ejes prioritarios que se concretarán -en función de cada territorio o sector estratégico- en algunas de las 86 categorías de gasto establecidas para el FEDER, el FSE y el Fondo de Cohesión. La distribución del número de ejes es la siguiente:

- FEDER, Regiones Convergencia, phasing-out y phasing-in': 7 ejes.

- FEDER, Regiones Competitividad regional y empleo: 5 ejes.

- FEDER, Regiones Ultraperiféricas, 2 ejes.

- FEDER, Cooperación transfronteriza, 5 ejes.

- Fondo Social Europeo: 5 ejes.

- Fondo de Cohesión: 3 ejes.

sus objetivos específicos, la participación de los Fondos Estructurales y los demás recursos financieros a aplicar. Se dividen en ejes prioritarios y se aplican mediante uno o más programas operativos (P.O.).

${ }^{6}$ Se denominan regiones phasing-in a las que entran en el objetivo de competitividad y empleo por convergencia natural; mientras que las regiones phasing-out son las que salen del objetivo convergencia por el efecto estadístico derivado de una UE ampliada frente a la UE-15. 


\begin{tabular}{|c|c|c|c|c|c|c|}
\hline & Fondo & $\begin{array}{c}\text { Regiones } \\
\text { Convergencia }\end{array}$ & $\begin{array}{l}\text { Regiones } \\
\text { Competitividad }\end{array}$ & $\begin{array}{l}\text { Regiones } \\
\text { Ultraperiféricas }\end{array}$ & $\begin{array}{l}\text { Cooperación } \\
\text { Transfronteriza }\end{array}$ & $\begin{array}{l}\text { Cooperación } \\
\text { Transnacional }\end{array}$ \\
\hline \multirow{4}{*}{$\begin{array}{l}\text { OE. 1: Convertir } \\
\text { a España en un } \\
\text { lugar más atrac- } \\
\text { tivo para invertir } \\
\text { y trabajar }\end{array}$} & \multirow{4}{*}{$\begin{array}{l}\text { FEDER / } \\
\text { Fondo } \\
\text { Cohesión }\end{array}$} & $\begin{array}{l}\text { Eje 3. FEDER: Medio } \\
\text { Ambiente, Entorno Na- } \\
\text { tural, Recursos Hídricos } \\
\text { y Prevención de Riesgos. } \\
\text { Eje 2. F. Cohesión: Medio } \\
\text { Ambiente }\end{array}$ & $\begin{array}{l}\text { Eje 2. FEDER: Medio } \\
\text { Ambiente y Prevención } \\
\text { de Riesgos y Energía. }\end{array}$ & Eje 1 y 2 RUP & $\begin{array}{l}\text { Eje 2. FEDER: } \\
\text { Medio Ambien- } \\
\text { te, Patrimonio y } \\
\text { Prevención de } \\
\text { Riesgos. }\end{array}$ & $\begin{array}{l}\text { Eje 2. FEDER: Medio } \\
\text { Ambiente }\end{array}$ \\
\hline & & $\begin{array}{l}\text { Eje 1. F. Cohesión: Re- } \\
\text { des transeuropeas de } \\
\text { transporte }\end{array}$ & $\begin{array}{l}\text { Eje 3. FEDER: Accesibi- } \\
\text { lidad a redes y servicios } \\
\text { de transporte }\end{array}$ & Eje 1 y 2 RUP & $\begin{array}{l}\text { Eje 3. FEDER: } \\
\text { Accesibilidad y } \\
\text { Ordenación del } \\
\text { Territorio. }\end{array}$ & $\begin{array}{l}\text { Eje 3. FEDER: } \\
\text { Accesibilidad }\end{array}$ \\
\hline & & $\begin{array}{l}\text { Eje 5. FEDER: Desarro- } \\
\text { llo Sostenible Local y } \\
\text { Urbano }\end{array}$ & $\begin{array}{l}\text { Eje 4. FEDER: Desarro- } \\
\text { llo Sostenible Local y } \\
\text { Urbano }\end{array}$ & & & $\begin{array}{l}\text { Eje 4. FEDER: Desarro- } \\
\text { llo Urbano Sostenible }\end{array}$ \\
\hline & & $\begin{array}{l}\text { Eje 6. FEDER: Infraestruc- } \\
\text { turas Sociales }\end{array}$ & & & & \\
\hline
\end{tabular}




\begin{tabular}{|c|c|c|c|c|c|}
\hline \multirow{3}{*}{$\begin{array}{l}\text { OE. 2: Mejorar } \\
\text { los conocimien- } \\
\text { tos y la innova- } \\
\text { ción a favor del } \\
\text { crecimiento }\end{array}$} & \multirow{2}{*}{ FEDER } & $\begin{array}{l}\text { Eje 1. Economía del } \\
\text { conocimiento }\end{array}$ & \multirow{2}{*}{$\begin{array}{l}\text { Eje 1. Economía del } \\
\text { Conocimiento, Inno- } \\
\text { vación y Desarrollo } \\
\text { Empresarial }\end{array}$} & \multirow{2}{*}{$\begin{array}{l}\text { Eje 1. Fomento de } \\
\text { la Competitividad } \\
\text { y la promoción } \\
\text { del empleo }\end{array}$} & \multirow{2}{*}{$\begin{array}{l}\text { Eje 1. Innovación y } \\
\text { Competitividad. }\end{array}$} \\
\hline & & $\begin{array}{l}\text { Eje 2. Desarrollo e } \\
\text { innovación }\end{array}$ & & & \\
\hline & FSE & \multicolumn{2}{|c|}{$\begin{array}{l}\text { Eje 1. Fomento del espíritu empresarial y mejora } \\
\text { de la adaptabilidad de trabajadores, empresas } \\
\text { y empresarios. }\end{array}$} & & \\
\hline \multirow{6}{*}{$\begin{array}{l}\text { OE. 3: Másy me- } \\
\text { jores empleos }\end{array}$} & \multirow[t]{2}{*}{ FSE } & $\begin{array}{l}\text { Eje 1. Fomento del espíri } \\
\text { de la adaptabilidad de } \\
\text { y empresarios. }\end{array}$ & $\begin{array}{l}\text { tu empresarial y mejora } \\
\text { trabajadores, empresas }\end{array}$ & \multirow{6}{*}{$\begin{array}{l}\text { Eje 4. Promover la cooperación } \\
\text { transnacional e interregional }\end{array}$} & \multirow[t]{2}{*}{$\begin{array}{l}\text { Eje 4. Promover la } \\
\text { cooperación transna- } \\
\text { cional e interregiona }\end{array}$} \\
\hline & & Eje 3. Aumento y mejora & del Capital Humano & & \\
\hline & \multirow{4}{*}{ FEDER } & \multicolumn{2}{|c|}{ Eje 1. Economía del Conocimiento } & & \\
\hline & & \multicolumn{2}{|c|}{ Eje 2. Desarrollo e innovación empresarial } & & \\
\hline & & \multicolumn{2}{|c|}{$\begin{array}{l}\text { Eje 3. Medio Ambiente, Entorno Natural, Recursos } \\
\text { Hídricos y Prevención de Riesgos }\end{array}$} & & \\
\hline & & \multicolumn{2}{|c|}{ Eje 5. Desarrollo Sostenible Local y Urbano } & & \\
\hline
\end{tabular}

Fuente: Dirección General de Fondos Europeos, 2007, Marco Estratégico Nacional de Referencia (MENR), 71 y 72. 


\section{Las regiones españolas y el objetivo de competitividad: análisis, metodología y algunos resultados}

Este apartado tiene como principal propósito comprobar desde un punto de vista cuantitativo la existencia o no de una orientación y apuesta de la política de cohesión de la UE hacia la mayor competitividad de sus territorios, a veces en menoscabo del principio de convergencia que la ha caracterizado desde sus orígenes. Para la realización de este objetivo respecto a las regiones españolas se analizarán los datos de programación y de ejecución del periodo 2007-2013, comparando los datos territoriales.

Los resultados de dicha comparación deberán, entonces, situarse en un contexto más amplio, presentando posteriormente, aunque sea de manera sucinta, cuál es la situación de las regiones europeas en relación con el objetivo de la competitividad, así como los retos a los que se enfrentan para alcanzarlo.

3.I. Medición del grado de ejecución de los principales programas operativos-FEDER en 2007-2015

Para el análisis del grado de ejecución de los programas operativos-FEDER ${ }^{7}$ se han utilizado los datos de programación y de ejecución de la Dirección General de Fondos Comunitarios para el período 2007-2013, dado que estos recogían las últimas reprogramaciones llevadas a cabo, difiriendo de los datos iniciales del MENR. Este periodo incluye el de programación propiamente dicho, 2007-2013, y el de ejecución, 2007-2015, atendiendo a la regla $n+2$. A pesar de que se disponía de la serie por año para todo el período, tanto en términos de ayuda como de gasto declarado, la comparación, debido a la irregularidad del proceso de ejecución anual, se basa en la suma total de todos los años, considerando el gasto total programado y la ejecución total, o gasto total ejecutado.

A partir de estos datos, el objetivo ha sido comprobar cuánto del gasto programado y de la ejecución van destinados a categorías de gasto ligadas a l+D+i con respecto al total de las 86 prioridades de gasto o temas de los Programas

\footnotetext{
7 El FEDER I+D+i hace referencia al denominado Fondo Tecnológico, cuya creación "para España se llevó a cabo durante la negociación de las perspectivas financieras 2007-2013, con el objetivo de que mejorara la inversión de las regiones españolas en esta área (en concreto de las empresas), y pudieran seguir la senda de crecimiento por la que había apostado la Política de Cohesión Europea" (Autor, 2013, p. 163).
} 
Operativos (POs). Para ello se han comparado las cantidades ejecutadas en I+D+i con respecto a las programadas -a esto se le ha denominado porcentaje de ejecución en $\mathrm{I}+\mathrm{D}+\mathrm{i}-$, haciendo lo mismo para el resto de los temas de los POs. Si el porcentaje de ejecución de la l+D+i es superior al del total del PO, se podrá concluir que no ha habido problemas en absorber los fondos destinados a la I+D+i que se programaron o reprogramaron inicialmente, o como mínimo, que fueron solventados a lo largo o al final de dicho período, de manera que incluso se han podido ver incrementados. Por el contrario, si este porcentaje de ejecución es menor, la conclusión sería justo la contraria y se podría convenir que el PO en cuestión ha tenido problemas para absorber determinada tipología de actuación.

\section{CUADRO 3. Categorías de gasto ligadas a I+D+i y a Pymes}

\begin{tabular}{|c|c|c|c|}
\hline \multicolumn{2}{|r|}{$1+D+i$} & \multicolumn{2}{|r|}{ Pymes } \\
\hline $\begin{array}{c}\text { Categorías } \\
\text { de gasto }\end{array}$ & Descripción & $\begin{array}{l}\text { Categorías } \\
\text { de gasto }\end{array}$ & Descripción \\
\hline 1 & Actividades de l+DT en centros de investigación & 6 & $\begin{array}{l}\text { Ayudas a las PYMEs para } \\
\text { el fomento de la utilización } \\
\text { de productos y procesos de } \\
\text { producción que respeten el } \\
\text { medio ambiente }\end{array}$ \\
\hline 2 & $\begin{array}{l}\text { Infraestructura de l+DT (instalaciones, instrumen- } \\
\text { tosy redes informáticas de alta velocidad para la } \\
\text { conexión de centros de investigación) y centros } \\
\text { de cualificación en una tecnología específica }\end{array}$ & 8 & $\begin{array}{l}\text { Otras inversiones en } \\
\text { empresas }\end{array}$ \\
\hline 3 & $\begin{array}{l}\text { Transferencia de tecnología y mejora de las } \\
\text { redes de cooperación entre pequeñas empresas } \\
\text { (PYME), así como entre éstas y otras empresas } \\
\text { y universidades, centros de enseñanza postse- } \\
\text { cundaria de todo tipo, autoridades regionales }\end{array}$ & 9 & $\begin{array}{l}\text { Otras medidas destinadas } \\
\text { a fomentar la investigación } \\
\text { e innovación y el espíritu } \\
\text { empresarial en las PYMEs }\end{array}$ \\
\hline 4 & $\begin{array}{l}\text { Ayudas para I+DT, en particular para las } \\
\text { PYME (incluido el acceso a servicios de I+DT } \\
\text { en centros de investigación) }\end{array}$ & & \\
\hline 5 & $\begin{array}{l}\text { Servicios avanzados de asistencia a empresas } \\
\text { y grupos de empresas }\end{array}$ & & \\
\hline 7 & $\begin{array}{l}\text { Inversión en empresas directamente relacio- } \\
\text { nadas con la investigación y la innovación } \\
\text { (tecnologías innovadoras, creación de nuevas } \\
\text { empresas por parte de las universidades, } \\
\text { centros y empresas de I+DT existentes, etc.) }\end{array}$ & & \\
\hline
\end{tabular}

Fuente: Elaboración propia a partir del Marco Estratégico Nacional de Referencia (MENR). 
Con respecto a las actuaciones vinculadas a la competitividad, para identificar las ligadas a la I+D+i se han elegido las categorías de gasto de los Programas Operativos 1, 2, 3, 4, 5 y 7 . Y también se han considerado las ligadas al apoyo a las PYMEs (6, 8 y 9), puesto que en muchos casos los procesos de innovación se encuentran incluidos, tal como se recoge en el Cuadro 3, donde se especifican los objetivos de gasto.

Los POs FEDER del período 2007-2013 son 19 + 4, es decir, 19 regionales (correspondientes a las 17 Comunidades Autónomas y a las dos Ciudades autónomas) más cuatro POs plurirregionales (Fondo de Cohesión, Asistencia Técnica, Fondo Tecnológico y Economía basada en el Conocimiento). En la Tabla 1 se puede ver desglosado el gasto programado y el ejecutado total para el período analizado por cada uno de los $23 \mathrm{PO}$ s, aunque por problemas de desagregación regional, especialmente en términos de gasto y ayuda declarada, no se tendrán en cuenta los programas plurirregionales. 
TABLA I. Gasto programado y ejecutado por tipología de Programas Operativos (PO) para el período 2007-2013

\begin{tabular}{|c|c|c|c|c|c|c|c|}
\hline & & \multicolumn{3}{|c|}{ Gasto programado } & \multicolumn{3}{|c|}{ Gasto ejecutado } \\
\hline & PO & $1+D+i$ & PYME & Total PO & $1+D+i$ & PYME & Total PO \\
\hline \multirow{5}{*}{ Convergencia } & Andalucía & 212.163 .865 & 1.584.088.649 & 8.554 .911 .768 & $314.428 .120,18$ & $1.662 .772 .771,68$ & $9.343 .528 .932,74$ \\
\hline & Castilla-La Mancha & 93.663 .989 & 290.776 .156 & 1.799.242.368 & $72.684 .846,65$ & $261.998 .380,89$ & 2.238.223.643.,54 \\
\hline & Extremadura & 67.086 .340 & 273.754 .320 & 1.975.234.952 & $30.142 .499,09$ & $301.913 .576,14$ & $2.061 .098 .027,19$ \\
\hline & Galicia & 118.412 .193 & 396.800 .100 & 2.739 .430 .504 & $80.711 .926,32$ & $325.637 .335,49$ & $2.866 .154 .750,92$ \\
\hline & & 491.326 .287 & 2.545 .419 .225 & 15.068 .819 .592 & 497.967.392 & 2.552.322.064 & 16.509 .005 .354 \\
\hline \multirow{5}{*}{ Phasing-out } & Asturias & 12.073 .115 & 94.523 .213 & 494.019 .011 & $8.940 .183,09$ & 96.316 .349 .94 & $570.109 .543,79$ \\
\hline & Ceuta & - & 10.018 .233 & 56.590 .780 & - & $9.020 .971,83$ & $60.366 .470,94$ \\
\hline & Melilla & 2.109 .378 & 7.905 .642 & 54.735 .637 & $2.156 .690,78$ & $6.695 .570,88$ & $60.254 .228,96$ \\
\hline & Murcia & 47.105 .099 & 127.683 .620 & 654.823 .841 & $50.718 .485,23$ & $140.186 .207,68$ & $729.222 .461,73$ \\
\hline & & 61.287 .592 & 240.130 .708 & 1.260 .169 .269 & 61.815 .359 & 252.219 .100 & 1.420 .052 .705 \\
\hline \multirow{4}{*}{ Phasing-in } & Castilla y León & 157.956 .200 & 253.410 .379 & 1.022 .743 .079 & $164.475 .806,26$ & $267.653 .187,72$ & $1.265 .376 .583,79$ \\
\hline & Comunidad Valenciana & 115.886 .266 & 376.051 .344 & 1.657 .925 .749 & $131.938 .939,94$ & $478.054 .576,91$ & $1.819 .396 .534,32$ \\
\hline & Islas Canarias & 75.669 .707 & 120.263 .026 & 1.602 .450 .159 & $53.963 .822,90$ & $133.536 .206,20$ & $1.690 .631 .724,46$ \\
\hline & & 349.512 .173 & 749.724 .749 & 4.283 .118 .987 & 250.378 .569 & 879.243 .971 & 4.775.404.843 \\
\hline
\end{tabular}




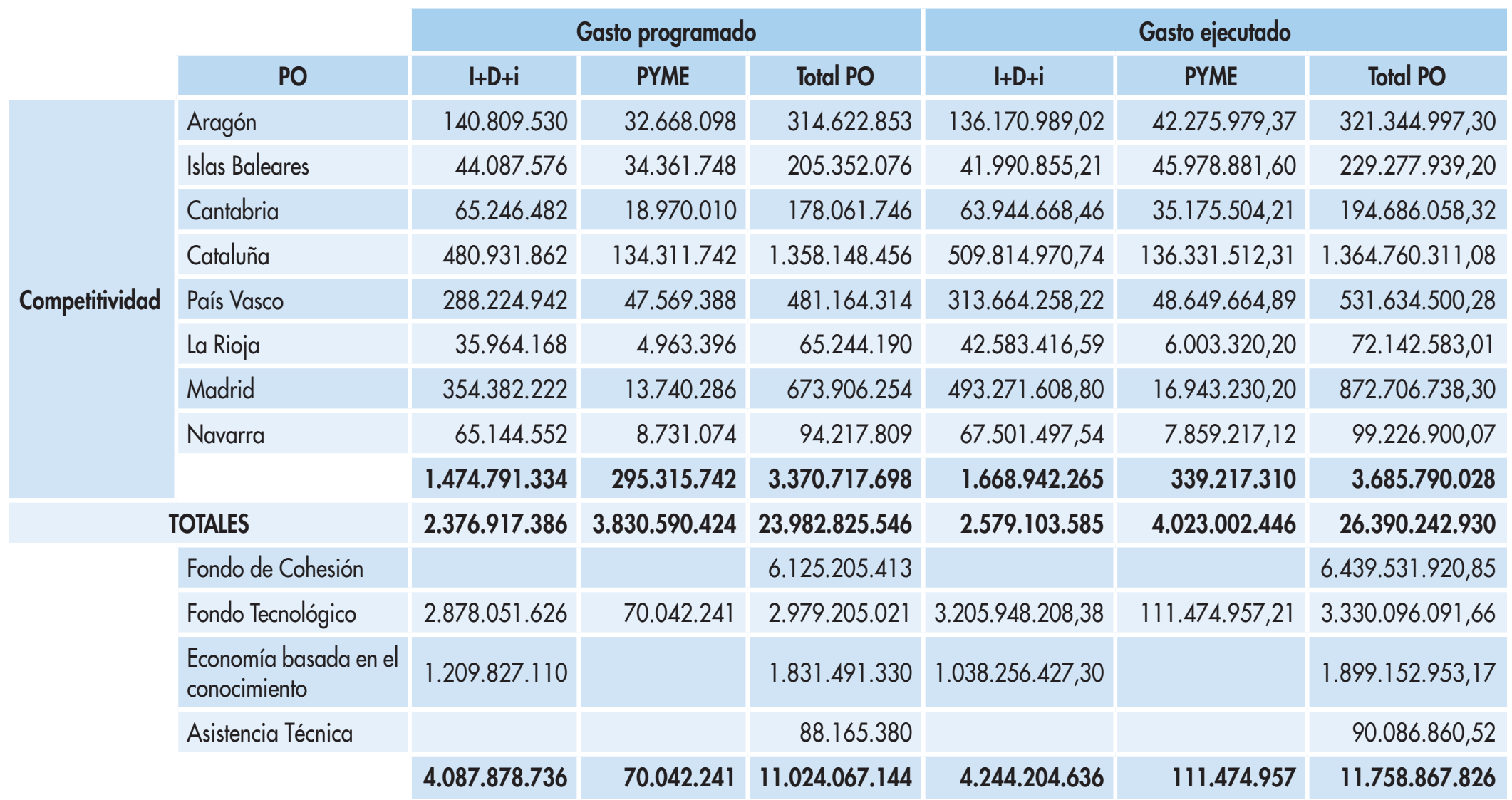

Fuente: Elaboración propia a partir de datos de programación y ejecución de la Dirección General de Fondos Comunitarios 2017. 


\section{TABLA 2. Porcentaje de ejecución por PO regional y tipología de región}

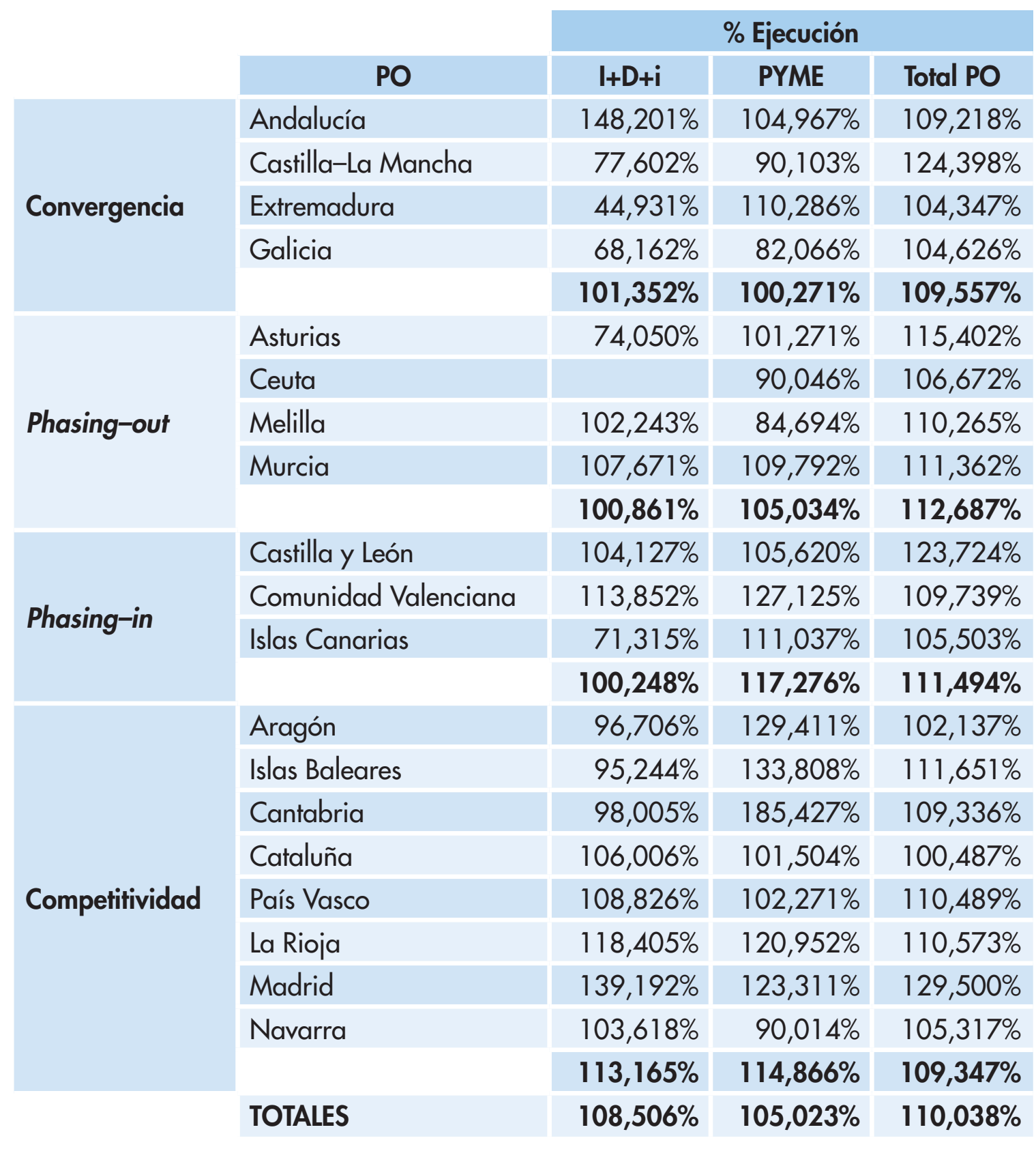

Fuente: Elaboración propia.

Si se calcula el porcentaje de ejecución tal y como se ha comentado, comparando el grado de ejecución en las categorías de gasto destinadas a la l+D+i con el grado de ejecución de todo el PO, se puede afirmar que, en conjunto, para todos 
los POs FEDER regionales del período 2007-2013, estas cifras son muy parecidas (en realidad la diferencia no llega a los dos puntos porcentuales: $-1,53$ entre lo destinado a l+D+i y el total, luego hay una leve infra ejecución en I+D+i). No hay que olvidar a la hora de analizar las cifras que durante el período completo ha habido muchos problemas de ejecución debido a la profunda crisis económica y financiera, y que lo que se está comparando son los datos de las últimas reprogramaciones con la ejecución final (Dirección General de Fondos Europeos 2009).

Aunque el porcentaje de gasto programado en I+D+i (lo mismo para PYME) en los $\mathrm{POs}$ regionales con respecto al total del PO coincide con el ejecutado $19,91 \%$ frente al 9,77\% respectivamente) -según se recoge en la Tabla 2-, lo cierto es que la distribución por tipología de región no ha sido uniforme y, para el período 2007-2013 la cuantía destinada a la competitividad ha estado en torno al 10\% (sin contar PYME). Tal como muestra la Figura 1, para la mayoría de los POs regionales que corresponden a regiones de convergencia, phasing-outy phasing-in, lo que se destina a l+D+i tiene un porcentaje de ejecución menor que el conjunto de los POs. Para los POs de las regiones de competitividad, donde claramente se ejecuta más I+D+i, hay todavía mayor diversidad entre las comunidades o regiones.

\section{FIGURA I. Diferencia entre el porcentaje de ejecución en I+D+i y el total del PO}

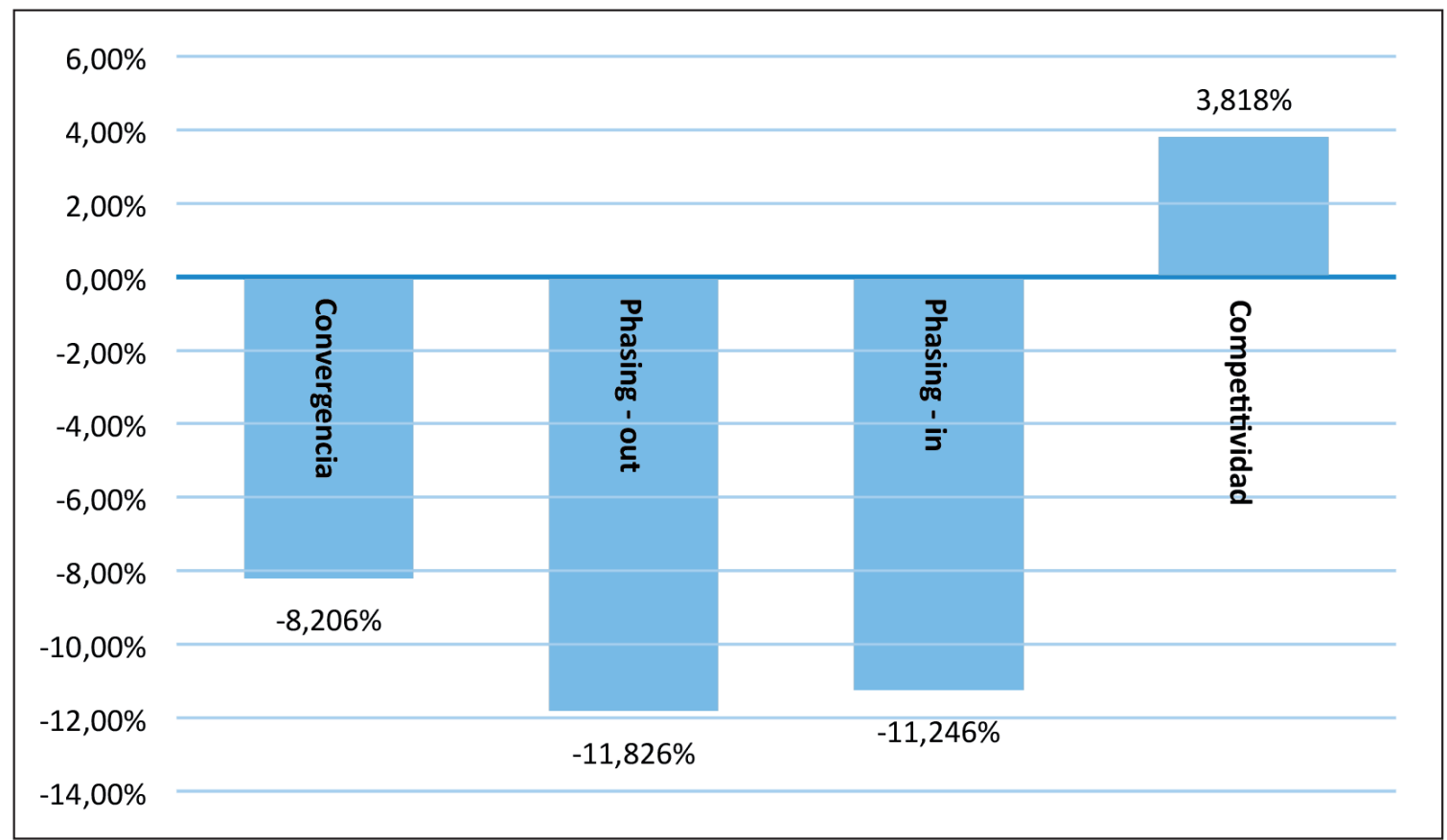

Fuente: Elaboración propia a partir de datos de la Dirección General de Fondos Comunitarios (2017). 
Se observa, por tanto, una evolución en las diferencias del grado de ejecución entre convergencia, phasing-out, phasing-in y competitividad. De hecho, las regiones convergentes no tienen diferencias tan negativas por la compensación tan grande de Andalucía. En esta Comunidad, pese a las dificultades iniciales, parece que el gasto en el período 2007-2013 acumula una cuantía importante, en buena parte porque no lo habían realizado en períodos anteriores, y quedaba pendiente (Dirección General de Fondos Europeos, 2013). En las regiones phasing-out ha habido más dificultades de absorción, especialmente para Ceuta, que no pudo ejecutar nada en I+D+i cuando sí lo había programado. Melilla y Murcia prácticamente han igualado las ratios de ejecución/programación en I+D+i y el resto de las categorías de gasto. Para las regiones phasing-in, salvo en la Comunidad Valenciana, tanto Castilla y León como Canarias tienen unas diferencias notables entre lo ejecutado en I+D+i y el resto del PO. Y el grupo de competitividad es el más heterogéneo: Cataluña, La Rioja y Madrid han ejecutado más I+D+i que el resto, pero el País Vasco y Navarra también están por encima de la media de ejecución.

\subsection{Los territorios españoles frente a la competitividad y la innovación}

Con el fin de continuar este estudio sobre los principios convergencia-cohesión y competitividad, el análisis del índice de competitividad se realizará según los distintos tipos de regiones que marca la política regional de cohesión, tal como se ha explicado en el punto anterior. Se han elegido los datos correspondientes al último índice publicado en 2016, porque recoge la situación de salida de las regiones tras el periodo analizado $2007-2015$.

El índice de competitividad está constituido por nueve factores agrupados en básicos (instituciones, estabilidad macroeconómica, infraestructura, salud y educación), eficientes (educación superior/formación continua, eficiencia del mercado laboral y tamaño del mercado) e innovadores (preparación tecnológica, complejidad empresarial e innovación). Ya lo afirmaban los autores en 2013, y sigue siendo válido para el último informe de 2016, "lo primero que destaca es la enorme disparidad en la distribución y en el peso que cada uno de ellos (factores) tiene en el resultado final, con variaciones destacadas, en algunos casos, entre los datos nacionales y los regionales" (García Nicolás, 2016, p. 189). La tabla 3 recoge las posiciones que ocupan las regiones españolas con respecto al conjunto de las 263 regiones analizadas, tanto atendiendo a cada uno de los grupos de factores como al índice global de competitividad. La región mejor situada es la Comunidad de Madrid tanto en el ICR como en el pilar de eficiencia y especialmente en el de innovación. La segunda mejor posición la ocupa el País Vasco, pero en un puesto 
mucho más alejado, aunque es la mejor situada con respecto al pilar de eficiencia. Por lo que respecta al pilar de factores básicos no hay ninguna región española situada entre las cien mejores. En el otro extremo del ranking se encuentran las dos Ciudades Autónomas de Ceuta y Melilla.

\section{TABLA 3. Posición global de las regiones españolas en el conjunto de la UE}

\begin{tabular}{|c|c|c|c|c|}
\hline & $\begin{array}{l}\text { Pilar } \\
\text { básico }\end{array}$ & $\begin{array}{l}\text { Pilar } \\
\text { eficiencia }\end{array}$ & $\begin{array}{c}\text { Pilar } \\
\text { innovación }\end{array}$ & $\begin{array}{l}\text { Índice de competitivi- } \\
\text { dad regional (ICR) }\end{array}$ \\
\hline Galicia & 181 & 191 & 179 & 181 \\
\hline Principado de Asturias & 173 & 188 & 172 & 174 \\
\hline Cantabria & 161 & 167 & 171 & 164 \\
\hline País Vasco & 153 & 78 & 134 & 119 \\
\hline Comunidad Foral de Navarra & 151 & 130 & 160 & 148 \\
\hline La Rioja & 154 & 196 & 185 & 184 \\
\hline Aragón & 140 & 194 & 169 & 173 \\
\hline Comunidad de Madrid & 104 & 82 & 57 & 83 \\
\hline Castilla y León & 152 & 199 & 192 & 187 \\
\hline Castilla-La Mancha & 143 & 240 & 207 & 216 \\
\hline Extremadura & 174 & 252 & 219 & 230 \\
\hline Cataluña & 129 & 175 & 138 & 153 \\
\hline Comunidad Valenciana & 156 & 205 & 175 & 184 \\
\hline Illes Balears & 167 & 222 & 178 & 200 \\
\hline Andalucía & 164 & 244 & 187 & 220 \\
\hline Región de Murcia & 160 & 227 & 196 & 210 \\
\hline Ciudad Autónoma de Ceuta & 190 & 261 & 236 & 238 \\
\hline Ciudad Autónoma de Melilla & 184 & 262 & 214 & 236 \\
\hline Canarias & 172 & 232 & 201 & 217 \\
\hline
\end{tabular}

Fuente: European Regional Competitiveness Index, 2016. 
A continuación, se muestran los factores que constituyen el Índice de competitividad regional para las regiones "convergencia" (Figura 2), regiones phasing-in y phasing-out (Figura 3), y regiones "competitividad" (Figura 4), siguiendo la clasificación de la política de cohesión para el periodo analizado. En estos casos se compara la región con las medias de España y de la UE. Del primer grupo, formado por Andalucía, Castilla-La Mancha, Galicia y Extremadura, es esta última la peor situada.

\section{FIGURA 2. Elementos del índice de competitividad en las regiones Convergencia (2016)}

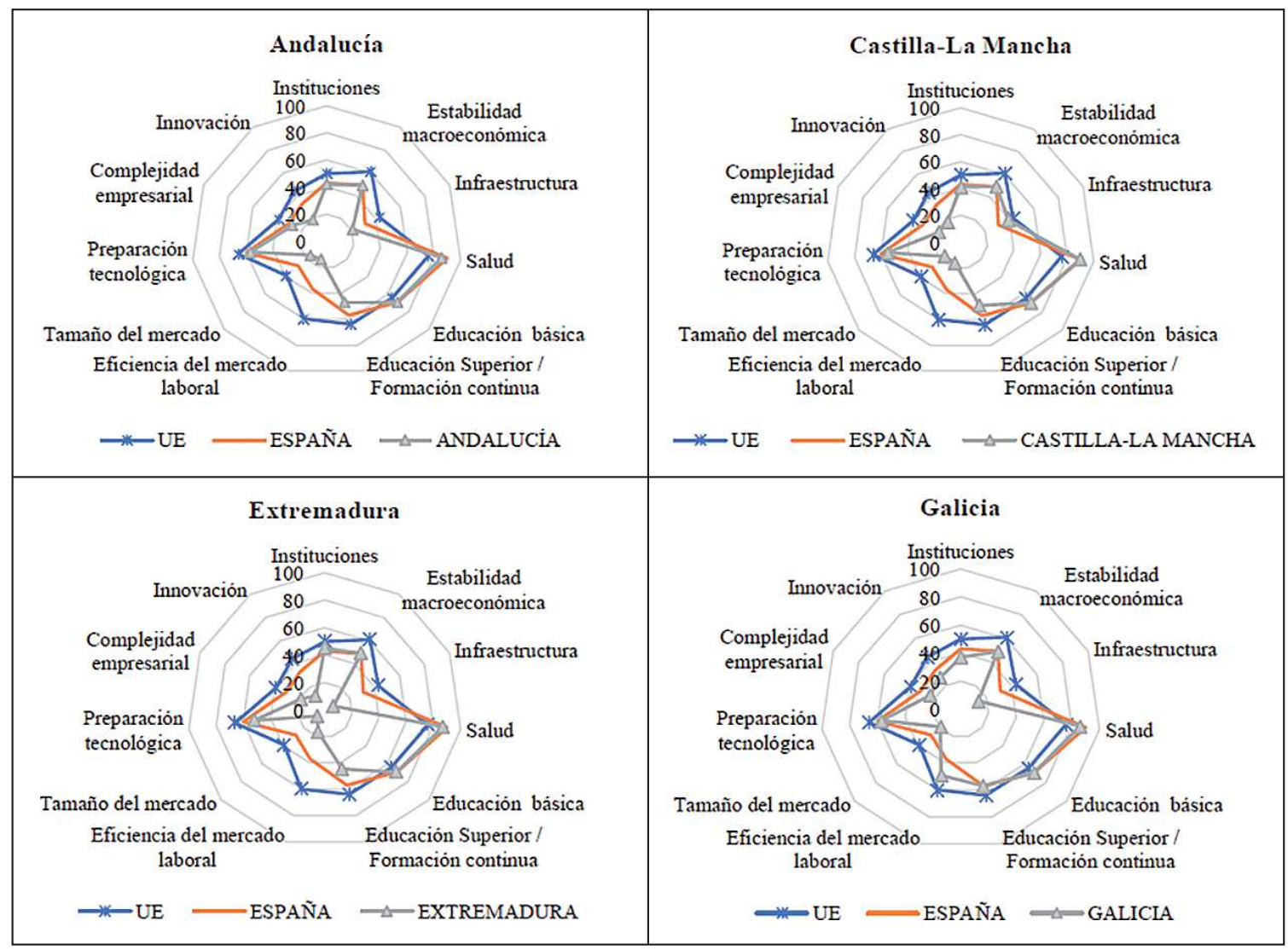

Fuente: Elaboración propia a partir de European Regional Competitiveness Index.

En el segundo grupo de regiones se distingue el caso extremo de Ceuta y Melilla con subíndices muy bajos en infraestructura, tamaño del mercado e innovación; Canarias, por debajo de la media nacional en todos los factores salvo eficiencia del mercado laboral, tamaño del mercado y complejidad empresarial. Del resto, 
Castilla y León, y la Comunidad Valenciana tienen unos niveles similares, mientras que Asturias y Murcia tienen un nivel en infraestructura por debajo de la media nacional. La Comunidad Valenciana y Asturias son las mejor situadas con respecto al factor innovación.

El tercer grupo de regiones es el más heterogéneo, aunque en general es el que ocupa las mejores posiciones, en muchos casos por encima de la media nacional e incluso de la europea.

Se puede completar el análisis de los distintos territorios con respecto a la competitividad, observando el índice de innovación. Para ello se ha utilizado el Regional Innovation Scoreboard 2017 que incluye la comparación con los datos de 2011 . El índice se construye a partir de cuatro tipos principales de indicadores -condiciones marco, inversiones, actividades de innovación e impactos ${ }^{8}-$ y diez dimensiones de innovación, de lo que resulta un total de veintisiete indicadores diferentes (European Commission, 2018).

\footnotetext{
${ }^{8}$ Las condiciones marco miden los principales motores del rendimiento en innovación externos a la empresa y abarcan tres dimensiones de innovación: recursos humanos, sistemas de investigación atractivos y entorno favorable a la innovación. El indicador «inversiones» mide la inversión pública y privada en la investigación y la innovación, y cubre dos dimensiones: financiación y apoyo e inversiones de las empresas. Las actividades de innovación tienen en cuenta los esfuerzos de innovación de la empresa agrupados en tres dimensiones de innovación: innovadores, vínculos y activos intelectuales. El indicador «impactos» abarca los efectos de las actividades innovadoras de la empresa en dos dimensiones de innovación: impactos en el empleo e impactos en las ventas.
} 


\section{FIGURA 3. Elementos del índice de competitividad en las regiones phasing-in y phasing-out}

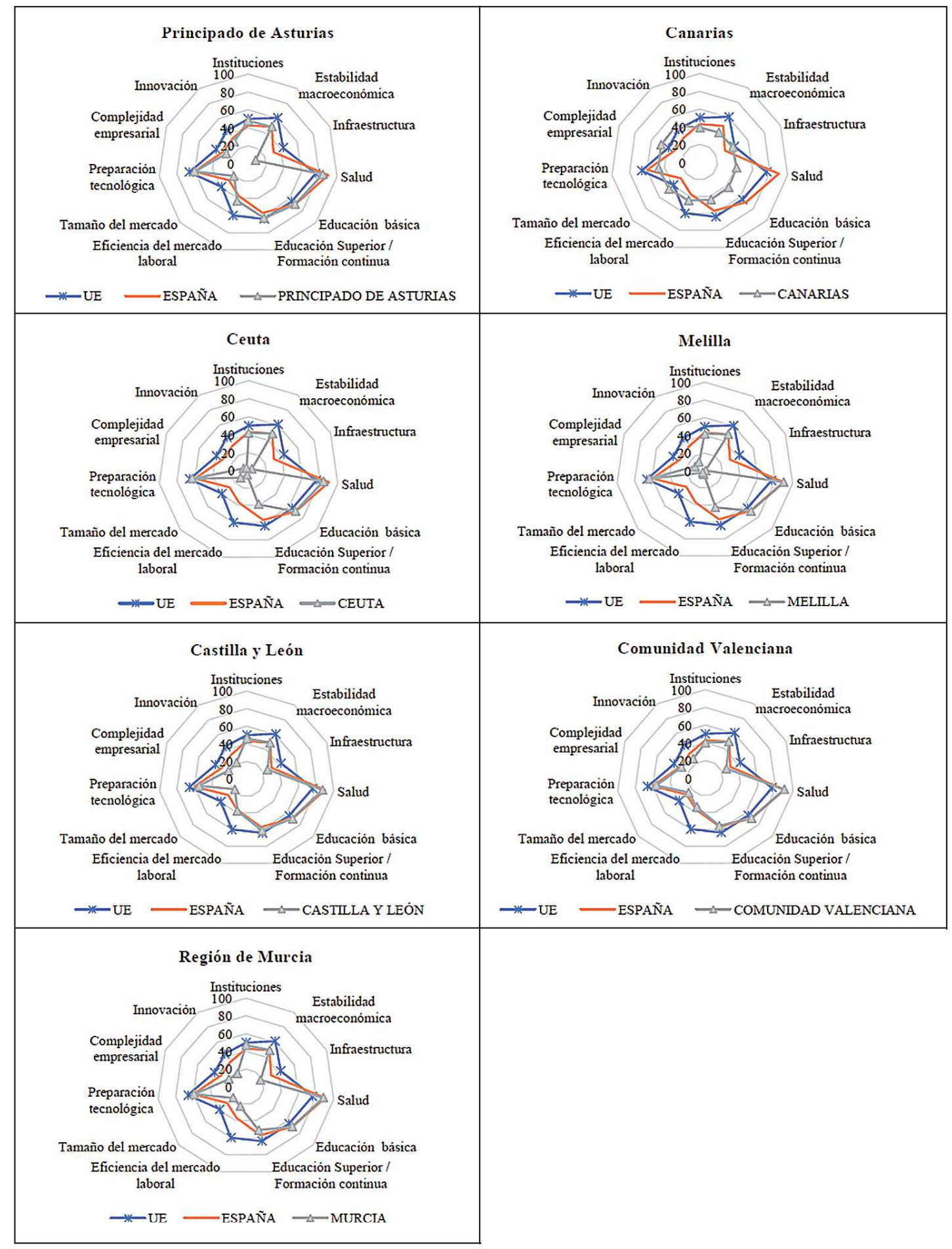

Fuente: Elaboración propia a partir de European Regional Competitiveness Index. 


\section{FIGURA 4. Elementos del índice de competitividad en las regiones competitividad}

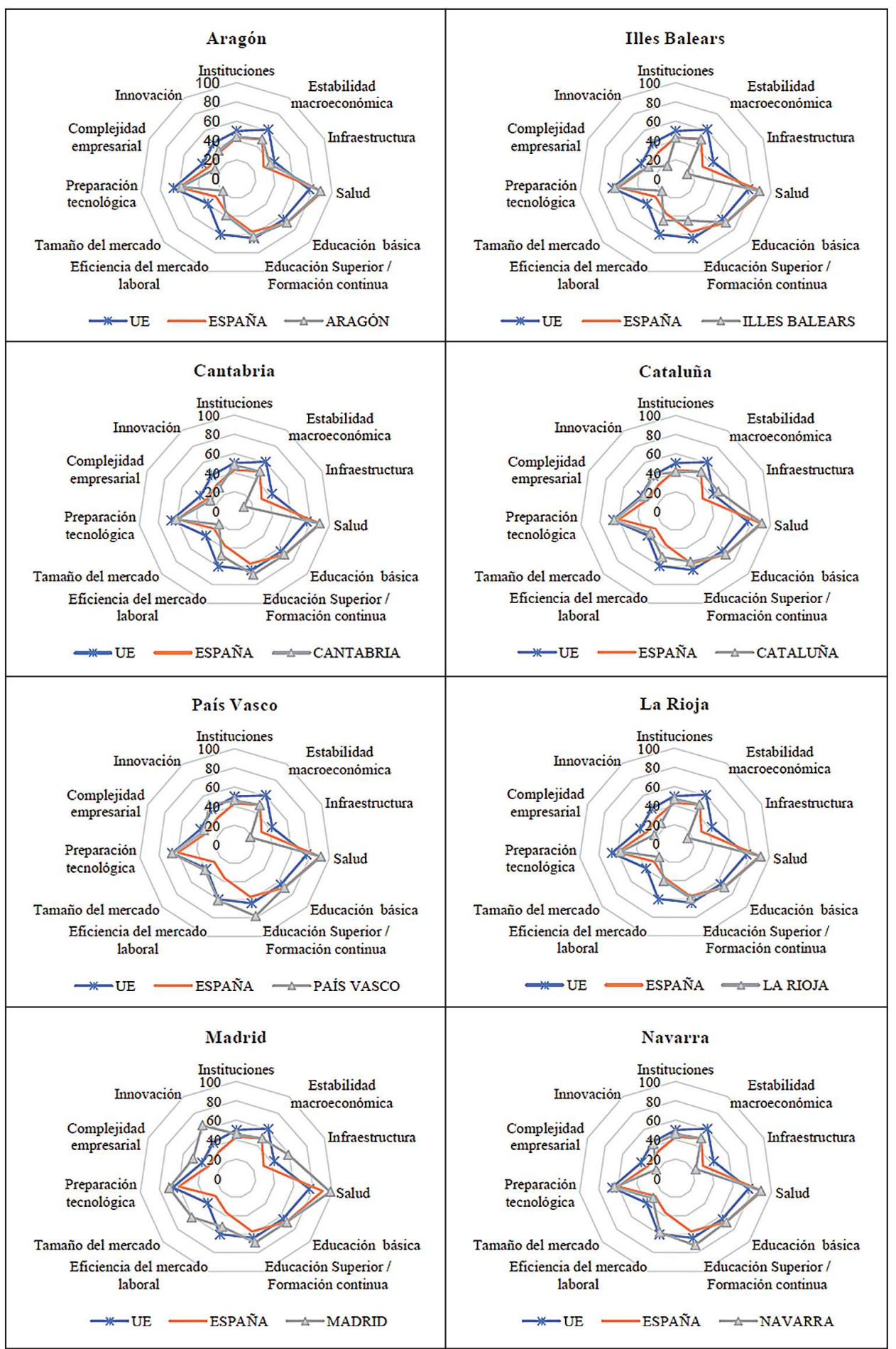

Fuente: Elaboración propia a partir de European Regional Competitiveness Index. 
El Gráfico 1 muestra la clasificación por Estados, situándose España en el grupo de los innovadores moderados que abarca las periferias sur $y$ oriental, aunque ha subido algunas posiciones desde el anterior índice. Cuando se desciende al ámbito regional, se observan grandes diferencias entre las distintas CCAA. En este caso es el País Vasco el territorio que destaca, considerado como innovador fuerte. El resto, salvo Canarias, aparecen clasificados como innovadores moderados.

\section{GRÁFICO I. Clasificación de los Estados Miembros de la UE, según el Índice europeo de Innovación 2018}

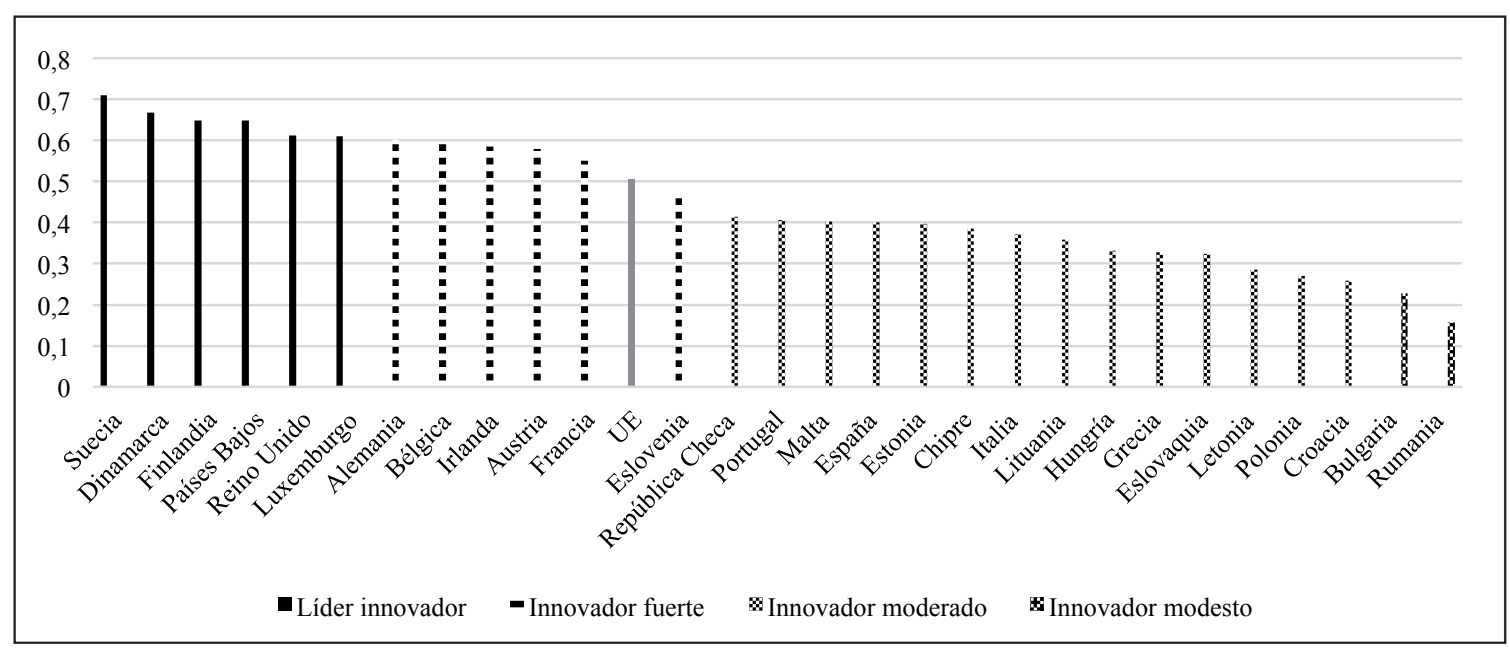

Fuente: European Innovation Scoreboard 2018 https://interactivetool.eu/f/extensions/DGGROW4/ EIS_2.html\#a

Las conclusiones de los dos índices estudiados coinciden -y en buena medida explican- con las debilidades regionales descritas por el propio Marco Estratégico Nacional de Referencia (MENR) para el período 2007-2013.

Tanto las regiones Convergencia como las phasing-out comparten debilidades relacionadas con "el bajo nivel formativo de la población y la elevada tasa de abandono escolar, con la existencia de empresas demasiado pequeñas para ser capaces de exportar e innovar, con una reducida inversión en I+D+i -sobre todo del sector privado- y con unas condiciones no adecuadas para el desarrollo de este tipo de inversión (débil colaboración entre los participantes en el ciclo de la innovación), además de una alta temporalidad en los contratos de trabajo, bajas tasas de empleo femenino y personas mayores de 55 años, y elevada tasa de paro de larga duración. Es de especial relevancia también, que en CCAA como Andalucía, Extremadura y Galicia la especialización productiva se realice en actividades de escaso valor añadido" (Murillo-García, 2013, p. 168). 
En el caso de las regiones Competitividad y phasing-in las debilidades tienen que ver con "baja utilización, en general, de las tecnologías asociadas a la sociedad de la información, insuficiente inversión en I+D+i -particularmente por parte de la iniciativa privada (excepto en Cataluña, La Rioja, País Vasco y Madrid)-, pérdida de competitividad (por costes y precios), concentración de los recursos humanos de calidad en determinadas zonas, e importante diferencia entre las tasa de empleo masculina y femenina. Además, en las regiones phasing-in, la actividad empresarial se concentra sectorialmente (Murillo-García, 2013, pp. 168-169).

\section{Algunas conclusiones y reflexiones finales}

A partir del breve repaso a la evolución del principio de convergencia y el objetivo de competitividad en la política europea de cohesión podemos apuntar algunas reflexiones generales sobre el contexto de competitividad.

Tanto la globalización como la crisis económica, en buena medida derivada de la primera, constituyen dos factores que han determinado el diseño de la política de cohesión en la última década. El paso de la convergencia a la competitividad, a partir de la Estrategia de Lisboa, supone también un cambio en la forma de abordar los problemas desde una perspectiva interna a una internacional. Mientras que en las primeras décadas hay una preocupación por la convergencia de los territorios europeos, el último decenio tiene como eje principal la posición de la UE con respecto a otros países y espacios económicos. Evidentemente, la globalización ha cambiado la escala a la que se ajustan los principios de las políticas de la UE, y, particularmente, de la política de cohesión. A ello es preciso añadir que la crisis económica hace mucho más evidentes las deficiencias internas (económicas, sociales e institucionales) para abordar grandes retos como el envejecimiento de la población, la inmigración, el cambio climático, etc.

Se observan, también, una serie de aspectos por resolver que afectan al desarrollo de la política de cohesión y del principio de convergencia, subrayados por Mancha y Gallo (2013, pp. 192-195): en primer lugar, la consecución de los objetivos de la Estrategia 2020 a través de la política de cohesión sin perder de vista que el principal objetivo de esta última es la reducción de las desigualdades; en segundo lugar, la condicionalidad macroeconómica ${ }^{9}$ en la recepción de los

\footnotetext{
${ }^{9}$ Artículo 23 (RDC). Medidas que vinculan la eficacia de los Fondos EIE a una buena gobernanza económica.
} 
fondos comunitarios; por último, la rígida gestión de éstos constituye también una dificultad, no sólo por los criterios de elegibilidad -que se reducen al PIB-, sino por la imposibilidad de trasvasar fondos entre Estados miembros en el caso de no utilización o de baja capacidad de absorción de los mismos.

Por lo que respecta al objetivo de nuestro trabajo podemos concluir que el análisis de ejecución de fondos destinados a l+D+i muestra una gran variedad de situaciones que coinciden en buena medida con la tipología de regiones a partir de la cual se aplican los fondos. Aún así existen también diferencias en el seno de los cuatro grupos regionales en que se divide el análisis: regiones competitividad, regiones convergencia, regiones phasing-out y regiones phasing-in.

La capacidad de absorción de los fondos para la competitividad y la innovación está estrechamente relacionada con la debilidad de algunas regiones en cuestiones como las infraestructuras, el mercado laboral o la salud de las instituciones. Las regiones competitividad son las que muestran una mayor capacidad de absorción de los fondos analizados, así como situaciones más favorables con respecto al objetivo de la competitividad. No obstante, quedan alejadas, como el resto de las regiones españolas, de la media europea en el índice de innovación.

Las diferencias entre las Comunidades Autónomas y de estas con el conjunto de la Unión Europea y del resto de territorios -tal como se recoge en el Índice de competitividad regional y en el de innovación- indican la necesidad de no abandonar el objetivo de la cohesión y sus fondos mientras se invierte en el fomento de la competitividad y de la innovación. No hacerlo incrementaría las divergencias territoriales tanto dentro de España como en el seno de la Unión Europea, y abocaría a algunas regiones a graves problemas económicos y sociales.

Las perspectivas financieras establecidas para el periodo 2014-2020 han reafirmado las tendencias observadas a nivel de programación para el periodo 2007-2013, tanto con respecto a España como al conjunto de la Unión Europea. Se ha consolidado claramente la competitividad como eje fundamental de la política regional comunitaria, apoyándose en la productividad, la formación, y los sistemas de I+D e innovación de unos territorios clasificados en regiones más

1. La Comisión podrá solicitar a un Estado miembro que revise y proponga modificaciones de su acuerdo de asociación y de los programas pertinentes cuando sea necesario para contribuir a la aplicación de recomendaciones pertinentes del Consejo o para maximizar el impacto de los Fondos EIE sobre el crecimiento y la competitividad en aquellos Estados miembros que reciban ayuda financiera. 
o menos desarrolladas. El Acuerdo de Asociación en España y la programación de los fondos de Cohesión para el periodo 2014-2020 recogen en su anexo de Recomendaciones la debilidad del Sistema español de Ciencia, Tecnología e Innovación, y la necesidad de concentrar los recursos en esa prioridad (Cordero Mestanza, 2015, p. 164).

\section{Referencias bibliográficas}

Annoni, P., Dijkstra, L. y Gargano, N. (2017). The EU Regional Competitiveness Index 2016. DG for Urban and Regional Policy, Working Papers WPO2/2017.

BARCA, F. (2009). An agenda for a reformed cohesion policy: A place-based approach to meeting European Union challenges and expectations. Independent Report prepared at the request of Danuta Hübner, Commissioner for Regional Policy, April 2009. Brussels: EU Commission.

Comisión de lAs COMUNIDAdes EUROPEAs (2005). Trabajando juntos por el crecimiento y el empleo. Relanzamiento de la estrategia de Lisboa COM(2005) 24 final. Comunicación del Presidente Barroso de común acuerdo con el Vicepresidente Verheugen $\{$ SEC(2005) 192\}\{SEC(2005) 193\}, Bruselas.

Comisión EUROPEA (2010a). Contribución de la Política Regional al crecimiento inteligente en el marco de Europa 2020, COM(2010) 553 final, SEC(2010) 1183, Bruselas.

- (2010b). EUROPA 2020. Una estrategia para un crecimiento inteligente, sostenible e integrador, $\operatorname{COM}(2010) 2020$ final, Bruselas.

- (2018). European Innovation Scoreboard 2018. Luxembourg: Publications Office of the European Union.

Consejo Europeo (2006). Decisión del Consejo de 6 de octubre de 2006 relativa a las directrices estratégicas comunitarias en materia de cohesión (2006/702/CE).

Cordero Mestanza, G. (2015). La promoción de la innovación en la política regional y de cohesión en el periodo 2014-2020: su aplicación en España. Investigaciones Regionales = Journal of Regional Research, n. ${ }^{\circ} 33$. Pp. 161-186. 
Dirección General de Fondos Comunitarios (2009). Informe 2009 de seguimiento estratégico del "Marco Estratégico Nacional de Referencia". Diciembre 2009, Ministerio de Economía y Hacienda, Madrid.

- (2007). Marco Estratégico Nacional de Referencia 2007-2013 (MENR), Ministerio de Hacienda, Madrid. http://www.dgfc.sepg.hacienda.gob.es/sitios/dgfc/ es-ES/ipr/fcp0713/p/menr/Paginas/inicio.aspx

- (2017). Informe de Ejecución MENR 2007-2013 (mimeo).

- (2013). Informe anual del Programa Operativo de Andalucía: www.dgfc. sepg.minhap.gob.es/sitios/dgfc/es-ES/ipr/fcp0713/gs/feder/ia/Documents/ INFORME_ejecucion_\%20\%202013_AN.pdf

EUROPEAN ReGIONALCOMPETTIIVENESSInDEX. https://ec.europa.eu/regional_policy/en/ information/maps/regional_competitiveness/

GarCía Nicolás, C. (2016): "La competitividad territorial y el Plan Europeo de Inversiones frente a la desigualdad regional", Investigaciones Regionales - Journal of Regional Research, n. ${ }^{\circ} 35$, Sección Política Regional Europea: pp. 177-201.

Garrido Yserte, R., Mancha Navarro, T. y Cuadrado Roura, J. R. (2007). La Política Regional y de Cohesión en la Unión Europea: veinte años de avance y un futuro nuevo. Investigaciones Regionales, n. ${ }^{\circ} 10$ : pp. 239-266.

JOUEN, M. (2011). La politique européenne de cohésion, La Documentation française, Paris.

KoK, W. (2004). Hacer frente al desafío: La Estrategia de Lisboa para el crecimiento y el empleo. Informe del Grupo de Alto Nivel presidido por Wim Kok, European Communities, Bruselas.

Mancha Navarro, T. y Gallo Rivera, T. (2013). Política regional y cohesión europea: perspectivas 2014-2020. Ekonomiaz, n. ${ }^{\circ}$ 82: pp. 170-199.

MurilLO-GARCíA, E. (2013): "¿Cómo han aplicado las CCAA la políitica de cohesión europea? Estrategias y retos de crecimiento, innovación y convergencia en la europa ampliada", en Colino, C. (coord.): Las comunidades autónomas frente a los retos de la nueva política de cohesión europea en la Europa ampliada. Capacidades y estrategias. Fundación Alternativas. Consejería de Hacienda y Sector público del Principado de Asturias, pp. 155-186. 
Parlement Europeen (2005). L'adaptation de la politique de cohésion à l'Europe élargie et aux objectifs de Lisbonne et de Göteborg, Direction générale Politiques internes de l'Union, Département thématique Politiques structurelles et de Cohésion, Développement régional, IP/REGI/ST/2004-008 30/05/2005, PE 350811.

UnIÓN EUROPEA (2004). Política Regional: «La cohesión a partir del viraje decisivo de 2007», Inforegio-ficha de síntesis 2004.

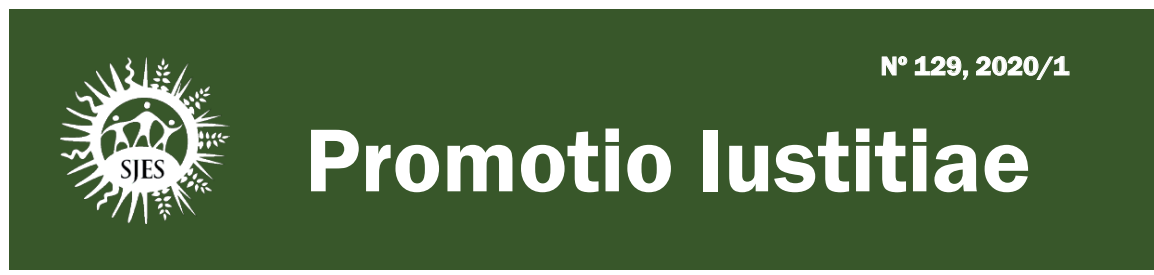

Secretariado para la Justicia Social y la Ecología (SJES), Curia General de la Compaña de Jesús, Roma = Italia

\section{Segundo Congreso de Apostolado Social}

Roma, 4 - 8 de noviembre de 2019
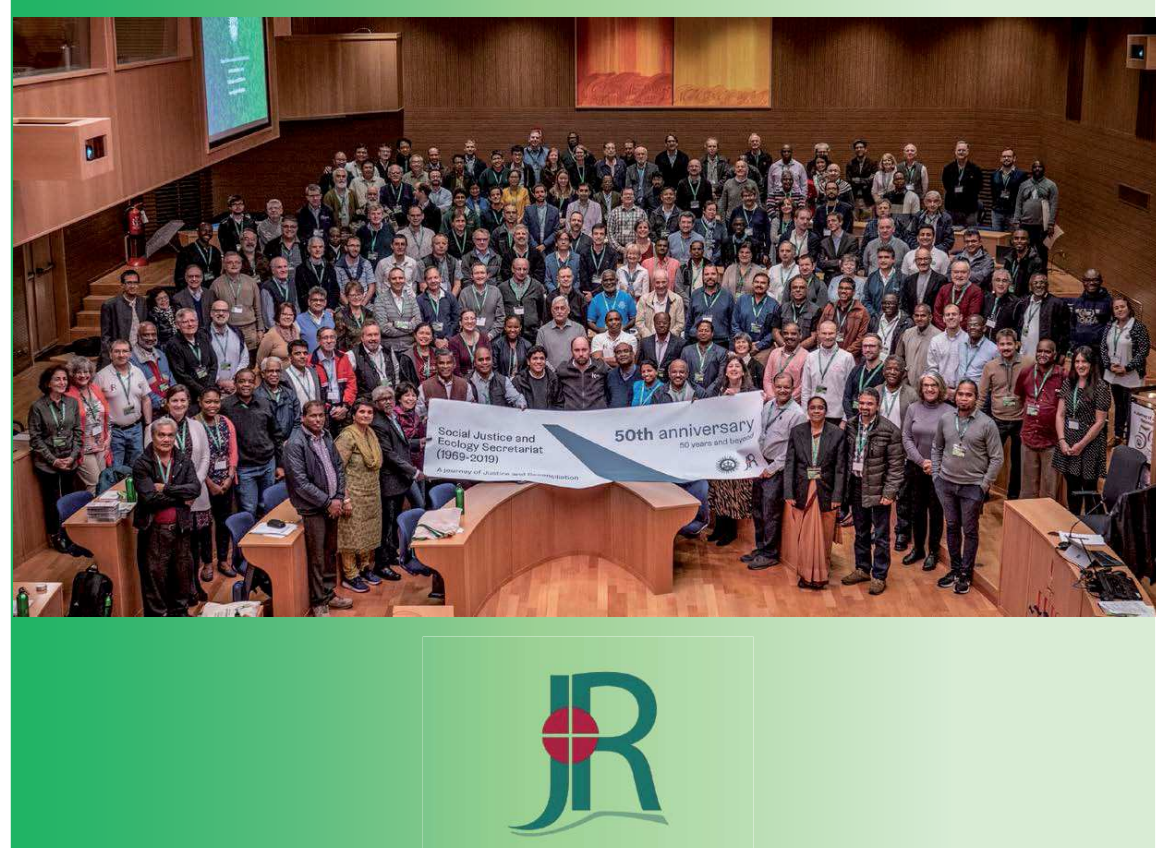